\title{
On the Kelvin-Helmholtz route to turbulence
}

\author{
S. A. Thorpe $\dagger$
}

School of Ocean Sciences, Bangor University, Menai Bridge, Anglesey LL59 5AB, UK

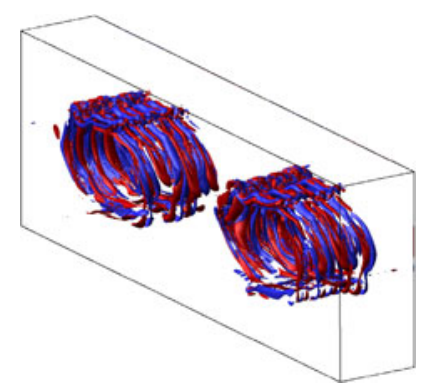

In their transition from a laminar state to turbulence, some unstable flows pass through a set of well-defined stages involving different and distinct processes. This is so, in particular, for Kelvin-Helmholtz instability, although details of its transition still retain many mysterious aspects. Billows develop in the primary stage of this stratified shear flow instability, separated by thin braids in which the shear is relatively high. Fluid is statically unstable within the billows and consequently potentially prone to convective instability. Numerical studies by Mashayek \& Peltier (J. Fluid Mech., this issue, vol 708, 2012a,b, pp. 5-44 and 45-70) have discovered several new types of secondary instability in the braids and billow cores that may hasten the eventual transition to turbulence. The instabilities are illustrated by the authors in colour figures, remarkable for their beauty and (recalling William Blake's 'The Tyger') 'fearful symmetry'. But are they helpful in establishing the subsequent turbulence in the natural environment?

Key words: stratified flows, transition to turbulence, turbulent mixing

\section{Introduction}

Kelvin-Helmholtz instability (KHI) is arguably the most elegant of the many types of instability found in fluids, and is widespread in its natural occurrence. Its name links two great scientists, Herman Helmholtz (sometimes Herman von Helmholtz) and Lord Kelvin (Sir William Thomson). They were friends. In summer, 1871, Helmholtz joined Kelvin on his schooner-yacht, the Lalla Rookh, to sail off Western Scotland. When Kelvin went ashore alone in Inveraray, he warned Helmholtz: 'Now, mind, Helmholtz, you're not to work at waves while I'm away' (Cahan 2012). Helmholtz had published a paper in 1868 which notes that undulations of increasing amplitude will grow on a sheet vortex and Kelvin, thinking of the generation of waves by wind, showed in 1871 that, even when a horizontal vortex sheet separates fluids of different density, the less dense above, sufficiently short waves will grow exponentially. Although both men consider only the instability of vortex sheets, the instability of flows (usually horizontal) with gradual changes in velocity and stable stratification is generically referred to as KHI.

G. I. Taylor took up the theoretical study of the instability in an essay for which he was awarded the 1915 Adams Prize of the University of Cambridge, work that

$\dagger$ Email address for correspondence: oss413@sos.bangor.ac.uk 
remained unpublished, 'hoping to be able to undertake experiments designed to verify, or otherwise, the results' until 1931 when he was persuaded to publish alongside a similar study by S. Goldstein. (The equation governing the flow's instability was subsequently called the 'Taylor-Goldstein equation' by Drazin in 1958.) Although Taylor tried ways to produce the instability in the laboratory, it appears that he overlooked the method of producing a stratified shear flow in a tilting tube used by Osborne Reynolds, a method subsequently adopted, first in an MSc study by Mittendorf (1961) and later by the present author (Thorpe 1971). (Dr Enzo Macagno kindly provided a copy of Mittendorf's thesis in 1967, and told me that G. I. Taylor had seen the tilting tube when he visited Iowa State University and had discussed the effect of it being tilted. He was presumably concerned about possible Rayleigh-Taylor instability - not observed - during the period of vertical acceleration.) A major advance was the proof by Howard and Miles in 1961 that, under a specific range of conditions (e.g. flows being horizontal, steady, inviscid and non-diffusive) stratified shear flows are stable to small disturbances if the gradient Richardson number is greater than $1 / 4$ everywhere.

The earliest numerical study of KHI was by Hazel (1972). He calculated growth rates and the initial form of the instability, but it was not until Patnaik, Sherman \& Corcos (1976) that two-dimensional (2D) finite-amplitude disturbances and billows, separated by braids, the first or primary stage of the transition from laminar to turbulent flow, were represented numerically. Although described in reports of earlier laboratory experiments, Corcos \& Sherman (1976) appear to be the first to analyse (in 2D) a 'secondary shear instability' on the tilted narrow braids between billows. A further important 2D secondary stage of instability is the amalgamation of pairs of billows, 'pairing' (or 'amalgamation instability'). By 1985 the first 3D secondary instability was recognized. Modelled by Klaassen \& Peltier (1985), it is a 'secondary convective instability' (SCI) resulting from static instability within the overturning billows. The locations of these and the new instabilities now known to occur in the KHI transition from laminar to turbulent flow are shown in figure 1.

\section{Overview}

There is a growing interest in KHI, most notably because it is one of the processes involved in the breaking of internal waves which contribute to turbulent diapycnal mixing. Many aspects of the instability are not yet understood, notably exactly how the transition from a laminar flow to turbulence takes place, and it is here that the numerical studies of Mashayek \& Peltier (2012b) (henceforth MP) make a substantial contribution. They examine billows growing in a flow with $\tanh z$ profiles of velocity and density ( $z$ being the vertical coordinate) in a range of initial Richardson, Reynolds and Prandtl ( $R i, R e$ and $P r$, respectively) numbers. MP discover four new types of three-dimensional secondary instabilities. Two appearing in or near the braids between billows are termed the 'stagnation point instability' and the 'secondary vorticity band instability'. The others occur within the billow cores. 'Localized core vortex instability' occurs near the ends of the braids. The other, termed the 'secondary core deformation instability', is oscillatory and tends to inflate the vortex cores of billows. MP compare the growth rates, buoyancy fluxes, energies and dissipation rates of the secondary instabilities as functions of time within $R i, R e$ and $P r$ space; the growth rates of secondary instabilities generally increase as $R e$ increases but decrease with increase in $R i$. They conclude that SCI is likely to be a key instability in the transition to 


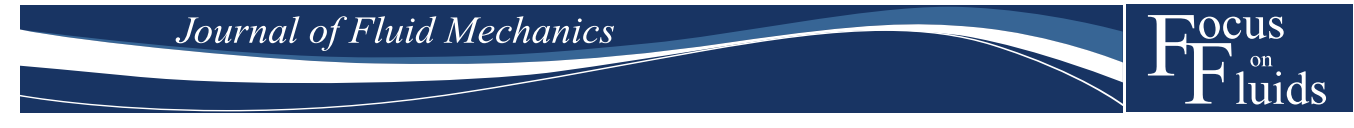

(a)

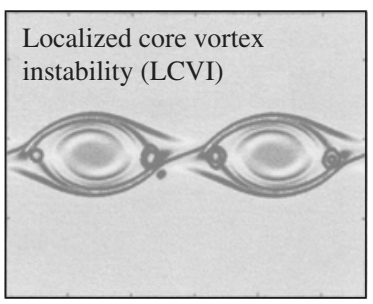

(d)

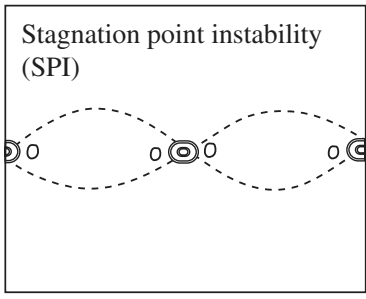

$(g)$

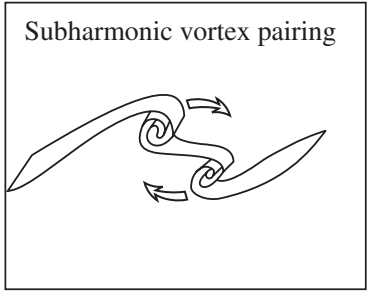

(b)

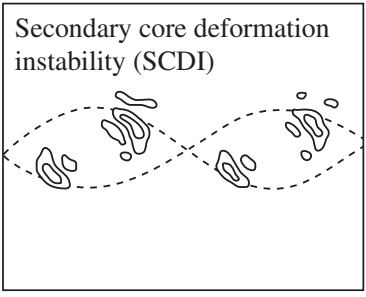

(e)

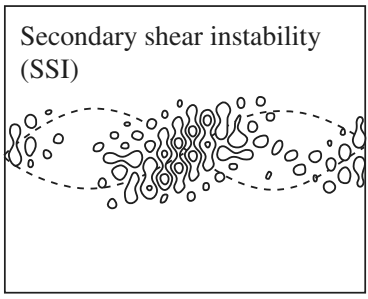

(h)

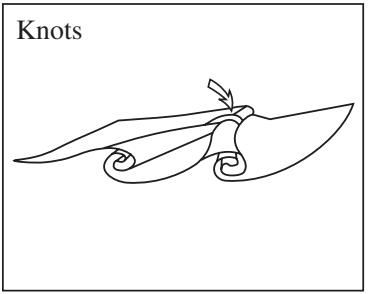

(c)
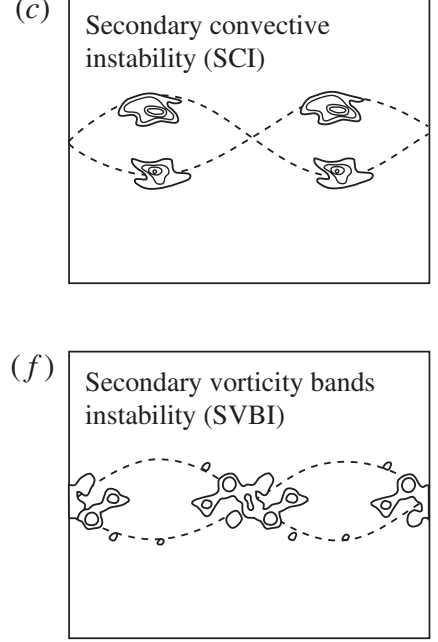

(i)

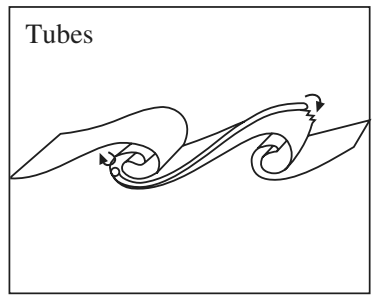

FIGURE 1. Stage 2 in the KHI transition from a parallel laminar flow to turbulence. Parts $(a-f)$ are described by MP; $(a),(b),(d)$ and $(f)$ represent the new instabilities they have discovered. Dashed lines indicate two billows with a braid between them, tilted from lower left to upper right, and full lines show secondary instabilities. The diagram is based on MP's lexicons of secondary instabilities, and on Thorpe (1987, figure 7) in which knot and tube instabilities (parts $h$ and $i$ ) are described. (I am grateful to my wife and Kate Davis for help with this figure.)

turbulence at high $\operatorname{Re}\left(O\left(10^{4}\right)\right)$ when $1<\operatorname{Pr}<8$. The new instabilities have not yet been reported in the laboratory and identification will be challenging.

\section{Future}

There still remain questions about just how the secondary instabilities lead to and affect turbulence. Do they impress on the (young) turbulent flow organized, if anisotropic, structures important in dissipation and mixing? Part of the justification of MP for examining the details of the secondary instabilities preceding turbulence is that their mixing is highly efficient and extends over a considerable period of the life-cycle of KHI. This justification appears sound, even though the time for which the structure of the initial billows and secondary instabilities are apparent is significantly less than the period in which small-scale structures occur and turbulence persists, before turbulence collapses back to a laminar flow state. MP also speculate that their study may help explain variations found in mixing efficiency in geophysical flows. 
MP conjecture that the interaction of two or more instability processes will mark a rapid transition to turbulence. They also ask: 'Does secondary instability suppress the tendency of billows to pair'? Pairing is commonly found in the laboratory, but is observed rarely, if ever, in the atmosphere or ocean. The answer seems to be: 'yes'. Mashayek \& Peltier (2011) find that when $R e \gtrsim 2000$, pairing is suppressed by the rapid growth of $3 \mathrm{D}$ secondary instabilities, as shown in the figure by the title ( $R e=6000, R i=0.12$ ). Numerical studies of the instability of long-crested billows, perhaps leading to the 'knots' observed in the laboratory (Thorpe 2002), where billows pair along their length (figure $1 h$ ), would provide useful measures of billow coherence and structure, and possibly estimates of extreme mixing. The conditions in which the numerical studies have been made and in which the Taylor-Goldstein equation is valid are, however, not those found in the generally high-Re conditions of the natural environment. There flow is unsteady and usually turbulent, if weakly so; the effective viscosity and diffusivity are non-zero and vary in $z$. Nevertheless, there are plentiful observations to show that billows do occur in the natural environment, indicating that structures can develop in turbulent flows very much as in the laminar flows described above. Some progress (Liu, Thorpe \& Smyth 2012) is being made on how pre-existing turbulence may affect the onset of instability. KHI leads to beautiful and still mysterious flow patterns. Its effects are yet to be fully revealed.

\section{References}

CAHAn, D. 2012 The awarding of the Copley Medal and the 'discovery' of the law of conservation of energy: Joule, Mayer and Helmholtz revisited. Notes Rec. R. Soc. 66, 125-139.

Corcos, G. \& Sherman, F. S. 1976 Vorticity concentration and the dynamics of the unstable free shear layers. J. Fluid Mech. 73, 241-264.

HAZEL, P. 1972 Numerical studies of the stability of inviscid stratified shear flows. J. Fluid Mech. 51, 39-61.

Klatassen, G. P. \& Peltier, W. R. 1985 The onset of turbulence in finite amplitude Kelvin-Helmholtz billows. J. Fluid Mech. 155, 1-35.

LiU, Z., Thorpe, S. A. \& SMYth, W. D. 2012 Instability and hydraulics of turbulent stratified shear flows. J. Fluid Mech. 695, 235-256.

Mashayek, A. \& Peltier, W. R. 2011 Three-dimensionalization of the stratified mixing layer at high Reynolds number. Phys. Fluids 23, 111701.

Mashayek, A. \& Peltier, W. R. $2012 a$ The 'zoo' of secondary instabilities precursory to stratified shear flow transition. Part 1 Shear aligned convection, pairing, and braid instabilities. J. Fluid Mech. 708, 5-44.

Mashayek, A. \& Peltier, W. R. $2012 b$ The 'zoo' of secondary instabilities precursory to stratified shear flow transition. Part 2 Effects of stratification. J. Fluid Mech. 708, 45-70.

Mittendorf, G. H. 1961 The instability of stratified flow. MSc thesis, Dept. of Mechanics \& Hydraulics, Iowa State University. 29pp.

Patnaik, P., Sherman, F. S. \& Corcos, G. M. 1976 A numerical simulation of Kelvin-Helmholtz waves of finite amplitude. J. Fluid Mech. 73, 215-240.

Thorpe, S. A. 1971 Experiments on the instability of stratified shear flows: miscible fluids. J. Fluid Mech. 46, 299-319.

Thorpe, S. A. 1987 Transitional phenomena and the development of turbulence in stratified fluids: a review. J. Geophys. Res. 92 (C5), 5231-5248.

Thorpe, S. A. 2002 The axial coherence of Kelvin-Helmholtz billows. Q. J. R. Meteorol. Soc. 128, $1529-1542$. 